\title{
Optimization of a perishable inventory system with both stochastic demand and supply: comparison of two scenario approaches
}

\author{
Duc Huy Nguyen ${ }^{1, *}$, Haoxun Chen ${ }^{1}$ \\ ${ }^{1}$ Laboratory of Industrial Systems Optimization, Charles Delaunay Institute \\ University of Technology of Troyes, 10004 Troyes Cedex, France \\ E-mail: 〈\{duc_huy.nguyen, haoxun.chen\}@utt.fr $\rangle$
}

\begin{abstract}
In this paper, we study a multi-period inventory model for a perishable product with both stochastic supply and demand in a rolling horizon framework. The product has a fixed shelf life such as fresh products, blood cells, chemicals, drugs and other pharmaceutical products. The objective is to minimize the expected total cost composed of ordering, purchasing, holding, shortage and waste costs. We focus on finding a high-quality solution close to the optimal solution of the model that provides decision support for decision-makers. We propose a stochastic programming model and transform it into MILP model based on conditional scenarios (CS) approach to reduce the computational burden. By comparing with the sample average approximation (SAA) method in a numerical study, we show that our method works efficiently.
\end{abstract}

Keywords: inventory management, optimization, perishable product, stochastic programming

Received: September 28, 2018; accepted: June 28, 2019; available online: July 4, 2019

DOI: $10.17535 /$ crorr.2019.0016

\section{Introduction}

According to the Collaborative Strategies to Reduce Unsaleables report [9], it is estimated that approximately $\$ 15$ billion of goods are wasted every year in the US food retail industry. This costs both manufacturers and retailers throughout their supply chains. Thus, it is vital to reduce the waste and improve the overall effectiveness of the supply chains. A significant proportion of the waste coming from product perishability is one of the major concerns for many industrial sectors such as fresh food, blood products, meat, chemicals, composite materials and pharmaceuticals. In most inventory models, it is assumed that products can be stored indefinitely to meet future demand. However, the effects of perishability cannot be ignored for certain types of products, which may become partially or entirely unsuitable for consumption as time passes $[1,5]$. Retail managers face significant challenges when unsold perishable items approach their expiration date. The decision-making becomes difficult due to the coexistence of items of several different ages. They have to choose among alternatives whether or not the remaining inventory should be sold at a price lower than expected or merely be considered as waste.

Besides, the existence of uncertainties in supply, delivery time, quality, and demand make the decision-making process more complicated. For most inventory models, the assumption of infinite supply may help it become less cumbersome, but this also reduces the model's precision. In reality, supply uncertainty can be found in three aspects: supply lead time, supply quantity and purchase price according to [8]. Thus, considering these uncertainties in inventory models has been proved to be critical for their real application in inventory management.

${ }^{*}$ Corresponding author. 
The rolling-horizon approach is usually used in a business environment that is characterized by unpredicted future uncertainty because planning decisions for far future could not be accurate enough. This approach can reduce the size of a model and lower computational complexity. For this reason, in this paper, we adopt the rolling horizon approach.

To the best of our knowledge, very few papers study inventory management of perishable products with both supply and demand uncertainty in a rolling horizon framework. For this reason, we investigate an inventory model with multi-periods for a perishable product with fixed life shelf under both stochastic demand and stochastic supply in a rolling horizon framework. Based on the product's remaining shelf life and some useful historical information related to demand and supply, we develop a practical approach to help retail managers in generating an effective inventory replenishment plan. The essential characteristics of the inventory model are perishability, stochastic demand, stochastic supply, and multi-period.

This paper includes several main contributions. Firstly, we propose a stochastic programming model for a perishable inventory system with fixed life shelf under both stochastic demand and stochastic supply in a rolling horizon framework. Secondly, we formulate a mixed integer linear programming (MILP) model equivalent to the original problem using the conditional scenarios (CS) approach. Thirdly, we conduct a numerical study to evaluate the performance of the proposed algorithm. The results show that our approach can find a high-quality solution with statistical performance guaranteed in a reasonable computation time. Sensitivity analysis is conducted to examine the effect of some critical parameters on the total system cost.

The remainder of the paper is organized as follows. In Section 2, the problem and its model are introduced. The two solution methods are presented in Section 3. A numerical study is performed and its results are analyzed in qtextsfSection 4 . Section 5 concludes this paper with some remarks for future research.

\section{Problem description and model formulation}

\subsection{Problem description}

In this paper, we consider a multi-period inventory problem for a perishable product with fixed shelf life in a rolling horizon framework. For simplicity, we assume that company uses the first in first out (FIFO) withdraw policy. In this paper, the order of events occurred in each period is given as follows. i) Fresh items arrive at the beginning of each period. Then, the inventory level of each age is updated. The expired items are discarded. ii) Based on the inventory policy used, an order then placed if necessary. After that, the demand and supply in this period are observed, and customer demand is satisfied as much as possible, and unsatisfied demand is lost sale. iii) At the end of each period, holding costs or shortage costs are charged based on the remaining inventory which will be carried over from the current period to the next. The out-dating costs are also charged for expired items.

\subsection{Model formulation}

In the following, we formulate a stochastic programming model for the inventory optimization problem of perishable product considered. The age of a product is denoted by $i \in\{1, \ldots, M\}$. Let $a, v, h, p w$ are unit ordering cost, unit purchasing cost, unit holding cost, unit shortage cost and unit waste cost for each unit of products expired, respectively. Let $I_{t}^{i}$ denote the inventory level of the product which has age $i$ at the end of period $t \in\{1, \ldots, T\}$. It is convenient to denote $I_{t}^{1}$ as fresh items of the product in stock at the end of period $t \in\{1, \ldots, T\}$.

In the first stage, the decision variable includes the timing of ordering $y_{t}$ and the ordering quantity $O_{t}$ in each period $t \in\{1, \ldots, T\}$. The second stage variables include the supply quantity

received $q_{t}$, inventory level $I_{t}^{i}$ for each age $i$, lost sales $B_{t}$ and outdated items $I_{t}^{M}$ at the end 
of period $t$. In the second stage, the recourse actions are made to correct the negative effects due to the first stage decision (the timing of ordering). In the model, all continuous decision variables are non-negative.

It is assumed that demand $D_{t}$ and supply $S_{t}$ follows a multivariate Gaussian distribution $N(\mu, \Sigma)$ where $\mu$ is a vector of mean of $\left\{D_{t}, S_{t}\right\}$ and $\Sigma$ is a covariance matrix that gives the correlation between the supply $S_{t}$ and the demand $D_{t}$. Note that $x^{+}=\max \{0, x\}$. The stochastic programming model for an inventory management of a perishable product is given as follows:

$$
\begin{aligned}
& (\mathbf{S P}) \quad \min \sum_{t=1}^{T} \mathbb{E}_{D_{t}, S_{t}}\left[a y_{t}+u q_{t}+h\left(\sum_{i=1}^{M-1} I_{t}^{i}\right)+p B_{t}\right. \\
& y_{t}=\left\{\begin{array}{cc}
1, & \text { if } O_{t}>0 \\
0, & \text { otherwise }
\end{array}=1, \ldots, T\right. \\
& Y_{t}^{i}=\left(D_{t}-\sum_{j=i}^{M-1} I_{t-1}^{j}\right)^{+} \quad i=1, \ldots, M, t=1, \ldots, T \\
& I_{t}^{i}=\left(I_{t-1}^{i-1}-Y_{t}^{i}\right)^{+}, \quad t=1, \ldots, T ; i=2, \ldots, M \\
& I_{t}^{1}=\left(q_{t}-Y_{t}^{1}\right)^{+}, \quad t=1, \ldots, T \\
& B_{t}=\sum_{i=1}^{M-1} I_{t-1}^{i}-\sum_{i=1}^{M} I_{t}^{i}+q_{t}-D_{t}, \quad t=1, \ldots, T \\
& q_{t}=\min \left\{S_{t}, O_{t}\right\}, \quad t=1, \ldots, T \\
& I_{t}^{i} \geq 0, \quad i=1, \ldots, M ; t=1, \ldots, T \\
& q_{t}, B_{t} \geq 0, y_{t} \in\{0,1\} \quad t=1, \ldots, T
\end{aligned}
$$

The objective in eq.(1) is to minimize the total expected cost which includes five types of costs: ordering, purchasing, inventory holding, lost sales and out-dating cost over the planning horizon of $T$ periods.

In eq.(2) the binary variable $y_{t}=1$ only if there is an order quantity $O_{t}$ in period $t$. Eq.(3) shows the variable $Y_{t}^{i}$ as the remaining demand after withdrawing all products having ages from $i$ to $M$ using FIFO withdraw policy. Note that the decision variables $Y_{t}^{i}$ are redundant, but they are introduced for the readability of the model. Eq.(4) and eq.(5) give the transition of inventory from the actual period to the next. In eq.(3) and eq.(4), we implicitly assume that for the first period, $t=1$, the variables $I_{t-1}^{i}$ are replaced by the predetermined parameters $I_{0}^{i}$ (initial inventory). Eq.(6) shows the inventory balance constraints between period $t$ and $t+1$. This equation also determines that lost sale, $B_{t}$, which only occurs when the supply quantity received, $q_{t}$, and the inventory of all age $i$ cannot meet demand, $D_{t}$. Eq.(7) ensures that the quantity received is always limited by the supply $S_{t}$.

\section{Solution approach}

In this section, the original model (SP) is transformed into an equivalent MILP model by applying the scenario approach. The sample average approximation (SAA) method is a conventional approach for stochastic optimization. Beltran-Royo proves that conditional scenarios (CS) method might provide a better solution than the SAA method [2]. That is why we develop a scenario-based optimization approach for solving our model based on conditional scenarios (CS) method. 


\subsection{Deterministic equivalent model}

Based on the scenario-based stochastic programming approach, we reformulate the model (SP) into a mixed integer linear programming (MILP) model. This approach allows to capture the uncertainty in an approximate way with the precision depending on the number of scenarios considered. Let $\omega \in \Omega$ be the index of a scenario (a possible realization of random variables) and its probability of occurrence is $p_{\omega}$. The mathematic expectation $\mathbb{E}($.$) in the objective function$ can be replaced by $\sum_{\omega} p_{\omega}[$.$] . Then, the nonlinear objective function in eq.(1) is transformed$ into the deterministic objective function eq.(10) The deterministic equivalent MILP model is given as follows:

$$
\begin{aligned}
& \min \sum_{t=1}^{T} a y_{t}+\sum_{\omega \in \Omega} \sum_{t=1}^{T} p_{\omega}\left[u q_{t, \omega}+h\left(\sum_{i=1}^{M-1} I_{t, \omega}^{i}\right)+p B_{t, \omega}+w I_{t, \omega}^{M}\right] \\
& \text { s.t. Constraints eq.(11) - eq.(20) }
\end{aligned}
$$

The index $\omega$ is added to each two-stage decision variables related to the scenario $\omega$ except for the ordering quantity $O_{t}$. The notation of the first-stage decision variables $y_{t}$ remains as before. The ordering quantity $O_{t}$ does not appear explicitly in the objective function but implicitly through the realization of random variables such demand $D_{t, \omega}$ and supply $S_{t, \omega}$. In the following, we linearize the nonlinear constraints given (2)-(6) of the original problem (SP). Note that $H$ is a big number. The constraint (2) can be linearized as follows:

$$
0 \leq O_{t} \leq H y_{t} \quad t=1, \ldots, T
$$

The eq.(3) can be linearized as follows:

$$
\begin{aligned}
& D_{t, \omega}-\sum_{j=i}^{M-1} I_{t-1, \omega}^{j} \leq Y_{t, \omega}^{i} \leq\left(D_{t, \omega}-\sum_{j=i}^{M-1} I_{t-1, \omega}^{j}\right)+\left(1-\alpha_{t, \omega}^{i}\right) H \\
& 0 \leq Y_{t, \omega}^{i} \leq \alpha_{t, \omega}^{i} H \\
& \alpha_{t, \omega}^{i} \in\{0,1\}
\end{aligned}
$$

That can be explained as follows:

i) if $\alpha_{t, \omega}^{i}=1$, then $Y_{t, \omega}^{i}=\left(D_{t, \omega}-\sum_{j=i}^{M-1} I_{t-1, \omega}^{j}\right) \geq 0$ due to eq.(12)- eq.(13)

ii) if $\alpha_{t, \omega}^{i}=0$, then $Y_{t, \omega}^{i}=0$ and $\left(D_{t, \omega}-\sum_{j=i}^{M-1} I_{t-1, \omega}^{j}\right) \leq 0$ due to due to eq.(12) - eq.(13)

The two cases of $\alpha_{t, \omega}^{i}$ imply that the constraint (3) can be replaced by the constraints (12)-(14) in the deterministic equivalent model.

Since the coefficients related to $I_{t, \omega}^{i} i=1, \ldots M$ in the objective function to minimize are positive, constraints (4) and (5) can be linearized as follows:

$$
\begin{aligned}
& I_{t, \omega}^{i} \geq\left(I_{t-1, \omega}^{i-1}-Y_{t, \omega}^{i}\right) \quad t=1, \ldots, T ; i=2 \ldots M \\
& I_{t, \omega}^{1} \geq\left(q_{t, \omega}-Y_{t, \omega}^{1}\right) \quad t=1, \ldots, T
\end{aligned}
$$

Constraint (6) ensure the inventory balance between period $t$ and $t+1$. Lost sale, $B_{t, \omega}$, occurs when the demand $D_{t, \omega}$ can not be satisfied in period $t$ under scenario $\omega$. The constraints are given by:

$$
B_{t, \omega}=\sum_{i=1}^{M-1} I_{t-1, \omega}^{i}-\sum_{i=1}^{M} I_{t, \omega}^{i}+q_{t, \omega}-D_{t, \omega}, \quad t=1, \ldots, T
$$


Constraint (7) specifies that the quantity received $q_{t, \omega}$ is the minimum between order quantity $O_{t}$ and a realization of supply capacity $S_{t, \omega}$ in period $t$ under scenario $\omega$. That constraint can be linearized as follows:

$$
\begin{aligned}
& S_{t, \omega}-\left(1-\gamma_{t, \omega}\right) H \leq q_{t, \omega} \leq S_{t, \omega} \\
& O_{t}-\gamma_{t, \omega} H \leq q_{t, \omega} \leq O_{t} \\
& \gamma_{t, \omega} \in\{0,1\}
\end{aligned}
$$

That can be explained as follows:

i) if $\gamma_{t, \omega}=1$, we have $q_{t, \omega}=S_{t, \omega}$ because $S_{t, \omega}$ is not only an upper bound but also a lower bound of $q_{t, \omega}$ due to constraint (18). We then have $q_{t, \omega}=S_{t, \omega} \leq O_{t}$ due to constraint (19). Then $q_{t, \omega}=\min \left\{S_{t, \omega}, O_{t}\right\}$.

ii) if $\gamma_{t, \omega}=0$. we have $q_{t, \omega}=O_{t}$ due to constraint (19) and $q_{t, \omega}=O_{t} \leq S_{t, \omega}$ due to constraint (18). So, $q_{t, \omega}=\min \left\{S_{t, \omega}, O_{t}\right\}$. Then in both cases, we have $q_{t, \omega}=\min \left\{S_{t, \omega}, O_{t}\right\}$.

\subsection{Conditional scenarios approach}

Since the computational complexity for solving the stochastic programming problem increases with the number of scenarios, some techniques to reduce the number of representative scenarios that should be considered: moment matching methods, the sample average approximation (SAA) method, approaches based on probability metrics among others. According to [2], the conditional scenarios (CS) problem allows to deal with uncertainty in shorter computational time, and it could yield a better solution. Also, the CS approach can be seen as an aggregation method, in which, aggregation weights are given by conditional probability function of the random parameters.

The notation for the conditional scenarios method is given in the following. Let $E$ and $e=1, \ldots, E$ be the number and the index of discrete realizations of random variable considered, respectively. Denote $r$ as the component index of random vectors, $r=1, \ldots, R$ and $\{r, e\}$ as the index pair for conditional scenarios. $\xi$ is random vectors including $R$ components $\left(\xi_{1}, \ldots, \xi_{R}\right)^{T}$. $\xi_{r}: r^{t h}$ is a component of random vector $\xi . \tilde{\xi}_{r, e}$ is a realization of random variable $\xi_{r}$ with $e=1, \ldots, E .\left\{\tilde{p}_{r, e}\right\}$ is the corresponding probability of the realization $\tilde{\xi}_{r, e}$. Denote $\hat{\xi}^{r, e}$ and $\left\{\hat{p}^{r, e}\right\}$ as a conditional scenario and its corresponding probability given at the end of the CS procedure, respectively. The conditional scenarios method [2] is given as follows:

1. Given a vector of random variables $\xi=\left(\xi_{1}, \ldots, \xi_{R}\right)^{T}$ with the index $r=1, \ldots, R$ and $\xi \sim N_{R}(\mu, \Sigma)$. For each random variable $\xi_{r}$ defined in a given interval $L_{r}$, we discretize it and obtain finite realizations $\tilde{\xi}_{r, e}, e=1, \ldots, E_{r}$ with the corresponding probabilities $\left\{\tilde{p}_{r, e}\right\} . E_{r}$ is the number of discrete realizations in the given interval $L_{r}=\left[a_{r}, b_{r}\right]$. For example, $L_{r}=\left[\mu_{r}-4 \sigma_{r}, \mu_{r}+4 \sigma_{r}\right]$.

(a) Divide the interval $L_{r}$ into $E_{r}$ sub-intervals of equal length $L_{r, e}=\left[a_{r, e}, b_{r, e}\right]$

(b) For each $L_{r, e}$, compute the discrete realizations, $\tilde{\xi}_{r, e}$ and their corresponding probabilities, $\tilde{p}_{r, e}$ as follows:

$$
\begin{aligned}
& \tilde{\xi}_{r, e}=\mathbb{E}\left[\xi_{r} \mid \xi_{r} \in L_{r, e}\right]=\mu_{r}+\sigma_{r} \frac{p d f\left(\alpha_{r, e}\right)-p d f\left(\beta_{r, e}\right)}{\operatorname{cdf}\left(\alpha_{r, e}\right)-\operatorname{cdf}\left(\beta_{r, e}\right)} \\
& \tilde{p}_{r, e}=\mathbb{P}\left(\xi_{r} \in L_{r, e}\right)=\operatorname{cdf}\left(\alpha_{r, e}\right)-c d f\left(\beta_{r, e}\right)
\end{aligned}
$$

where $\alpha_{r, e}=\left(a_{r, e}-\mu_{r}\right) / \sigma_{r}$ and $\beta_{r, e}=\left(b_{r, e}-\mu_{r}\right) / \sigma_{r}$. Let $p d f$ and $c d f$ be the probability density function and the cumulative distribution funtion, respectively, of a standard normal variable. Let $\mu_{r}$ and $\sigma_{r}$ be the mean and the standard deviation of the random variable $\xi_{r}$, respectively. 
2. We compute the conditional scenarios $\hat{\xi}^{r, e}$ and the corresponding probabilities $\left\{\hat{p}^{r, e}\right\}$ by using the discrete realizations, $\tilde{\xi}_{r, e}$ found in step 1 as follows:

$$
\hat{\xi}^{r, e}=\mathbb{E}\left[\xi \mid \tilde{\xi}_{r, e}\right]=\mu+\frac{\tilde{\xi}_{r, e}-\mu_{r}}{\sigma_{r}^{2}} \Sigma_{r} \quad \text { and } \quad \hat{p}^{r, e}=\tilde{p}_{r, e}
$$

The total number of conditional scenarios is: $\sum_{r=1}^{R} E_{r}$ where $E_{r}$ is the number discrete realizations of $\xi_{r}$ into $\tilde{\xi}_{r}$.

The above procedure can be summarized by the following procedure: $\xi \rightarrow\left\{\tilde{\xi}_{r, e}\right\} \rightarrow\left\{\hat{\xi}^{r, e}\right\}$. Note that the index pair for conditional scenario $\{r, e\}$ and its corresponding probability are replaced by the index for scenario $\omega$ and $p_{\omega}$ in the subsection 3.1 .

\subsection{Solution evaluation}

To evaluate the solution quality, we compare our approach with the most common approach, sample average approximation (SAA) method. In this technique, the expected objective function of the stochastic problem is approximated by an average sample estimate derived from a random sample.

The main difference between SAA and CS approach is the way to approximate the random vector $\xi$ and their associated probabilities (equal probability versus conditional probability). Besides, only one sampling is made in CS approach, but a large number of samplings is necessary to ensure the quality of solution in SAA approach. In addition, the use of reduction techniques such as Latin hypercube sampling [6,4] could increase the efficiency of the SAA method. For this reason, we applied this technique to generate the whole scenarios in the SAA method.

\section{Numerical study}

In this section, numerical studies are conducted to evaluate the performance of the proposed algorithm. All MILP problems related to CS and SAA approach were solved by a solver Gurobi 6.5 with default parameters on a HP computer with Intel Core i5-4210M CPU $2.66 \mathrm{GHz}$ and 8.0 GB RAM.

\subsection{Data generation}

In the numerical study, we use the data from $[3,7]$ such as the fixed maximum shelf life, average demand, coefficient of variation $(C V=\sigma / \mu)$, number of period. By following [3], we normalize all cost parameters on the purchasing cost $v$, which is set to 1 . The unit holding cost ratio $h / v$ is varied from 0.02 to 0.04 . The unit lost sales cost $p$ is determined from the lost sales cost ratio $p /(v+p+h)$, which is varied between $90 \%$ and $99 \%$. The unit out-dating cost ratio, $w / v$ is varied between 0 and 1 . The fixed ordering cost for each order placed is varies from $250 v$ to $500 v$. The maximum shelf life is $M=2$ or 3 . The number of periods is $T=7$. The mean demand for each period is computed by $\mu_{D_{t}}=f_{t} \cdot \mu_{D}$ where the demand pattern in each period is $f_{t}=\{0.12,0.13,0.13,0.16,0.18,0.18,0.10\}$, and the value of mean demand in each period $\mu_{D_{t}}=[240,260,260,320,360,360,200]$ and $\sigma_{D_{t}}=0.25 \mu_{D_{t}}$. Supply follows a stationary normal distribution with $\mu_{S_{t}}=320$ and $\sigma_{S_{t}}=0.2 \mu_{S_{t}}$ for all $t=1, \ldots, 7$. 


\subsection{Performance evaluation of the solution approach}

In this section, we present our computational experiments on the algorithms proposed in the previous section for solving instances. To formulate the CS problem, we apply Section 4.1 to calculate the conditional scenarios that approximate the normal random vector which includes $\mu=\left\{\mu_{D_{t}}, \mu_{S_{t}}\right\}$ and $\sigma=\left\{\sigma_{D_{t}}, \sigma_{S_{t}}\right\}$. For $T=7$, we have $R=14$ random parameters, $E=12$ discretization points and $L_{r}=\left[\mu_{r}-4 \sigma_{r}, \mu_{r}+4 \sigma_{r}\right]$. The choice of $E=12$ can be explained as follows: we considered the (MILP) problem with different discretization level such as $E=$ $5,6,7 \ldots, 100$. We observe that the scenarios optimal solution has very similar quality compared with others for the discretization with $E \geq 12$.

In fact, the SAA and CS approaches try to solve the original problem approximately with an intention to reduce the computational burden. However, it is hard to assess the quality of the optimal SAA and CS solutions by using the optimality gap because we can not find the optimal solution of the original problem. In this case, we assess the E-SAA and E-CS value which are the expected value of the objective function of the original problem obtained at the SAA solution and CS solution, respectively. We generate randomly 1000000 sampling from a multivariate normal distribution of $\xi=\left\{D_{t}, S_{t}\right\}, t=1, \ldots, T$ to calculate the value E-SAA and E-CS.

Table 1 and Table 2 present the computational time and solution obtained by CS and SAA approach. All instances include 4368 continuous variables, 3192 binary variables, and 16471 constraints. The $9^{\text {th }}$ and $14^{\text {th }}$ columns show the relative gap between the two solutions found by CS and SAA approach, (E-SAA - E-CS)/E-CS, in two case $M=2$ and 3. Based on the inferential statistical analysis, the obtained solutions, E-SAA, could achieve an approximatively optimal solution with probability $95 \%$ within $0.1 \%$ deviation from the true optimal solution. The CS approach performs efficiently since the E-CS value is always less than the E-SAA value with $0.07 \%$ and $0.09 \%$ in average for $M=2$ and 3 , respectively. Besides, the CS approach has much shorter CPU times (35.37s versus $284.91 \mathrm{~s}$ and $35.41 \mathrm{~s}$ versus $317.08 \mathrm{~s}$ respectively). The numerical study suggests that the CS approach has a good balance between the solution quality and the computational time.

\subsection{Analysis of the solution}

In this study, we consider a perishable product with the fixed shelf life $M=2$. The ordering cost, unit holding cost, unit shortage cost, and unit wastage cost are $\$ 30, \$ 2, \$ 15, \$ 3$ respectively. The parameters of a distribution of demand and supply are given as follows: $\mu_{D}=$ $[860,810,760,570,620] ; \mu_{S}=[520,550,860,940,760] ; \sigma_{D}^{2}=[29584,26244,23104,12996,15376] ;$ $\sigma_{S}^{2}=[10816,12100,29584,35344,23104]$.

Figure 1 shows the cost distribution of the base case for the perishable inventory system over planning horizon $T=5$. The optimal value of the total expected cost of CS problem is $\$ 41740,98$.

As we can see, the purchase cost and shortage cost are the primary cost drivers that account for nearly $71 \%$ and $27 \%$ of the total system costs respectively. The holding cost is only $1 \%$, so in this case, the storage of a significant amount of product seems not critical. This result shows the important of inventory policy on the total system cost because it affects the purchased quantity and unmet demand strongly. 


\begin{tabular}{|c|c|c|c|c|c|c|c|c|}
\hline \multirow{3}{*}{$\mathrm{a}$} & \multirow[b]{3}{*}{$\mathrm{h}$} & \multirow[b]{3}{*}{$\mathrm{p}$} & \multirow[b]{3}{*}{$\mathrm{w}$} & \multicolumn{5}{|c|}{$\mathrm{M}=2$} \\
\hline & & & & \multicolumn{2}{|c|}{ CS approach } & \multicolumn{2}{|c|}{ SAA Approach } & \multirow[b]{2}{*}{$\%$} \\
\hline & & & & CPUs & E-CS & CPUs & E-SAA & \\
\hline 250 & 0.02 & 10 & 0.5 & 41.70 & 4292.30 & 412.22 & 4296.62 & $0.10 \%$ \\
\hline 250 & 0.02 & 10 & 1 & 46.02 & 4314.66 & 416.08 & 4320.61 & $0.14 \%$ \\
\hline 250 & 0.02 & 20 & 0.5 & 37.08 & 4624.11 & 277.45 & 4627.32 & $.07 \%$ \\
\hline 250 & 0.02 & 20 & 1 & 42.38 & 4650.57 & 278.18 & 4651.37 & $.02 \%$ \\
\hline 250 & 0.02 & 100 & 0.5 & 21.74 & 7168.08 & 124.52 & 7165.30 & $.04 \%$ \\
\hline 250 & 0.02 & 100 & 1 & 22.70 & 7187.49 & 124.44 & 7189.40 & $03 \%$ \\
\hline 250 & 0.04 & 10 & 0.5 & 50.63 & 4310.32 & 402.67 & 4316.87 & $15 \%$ \\
\hline 250 & 0.04 & 10 & 1 & 50.46 & 4335.91 & 404.42 & 4340.85 & $11 \%$ \\
\hline 250 & 0.04 & 20 & 0.5 & 44.42 & 4643.95 & 284.44 & 4648.22 & $09 \%$ \\
\hline 250 & 0.04 & 20 & 1 & 37.32 & 4672.40 & 284.28 & 4672.30 & $00 \%$ \\
\hline 250 & 0.04 & 100 & 0.5 & 20.13 & 7190.01 & 131.28 & 7187.21 & $04 \%$ \\
\hline 250 & 0.04 & 100 & 1 & 21.31 & 7208.03 & 137.75 & 7211.31 & $05 \%$ \\
\hline 500 & 0.02 & 10 & 0.5 & 40.72 & 13 & 493.51 & 6046.23 & $02 \%$ \\
\hline 500 & 0.02 & 10 & 1 & 45.74 & 60 & 471.14 & 6070.21 & $08 \%$ \\
\hline 500 & 0.02 & 20 & 0.5 & 35.96 & 6376.24 & 281.32 & 6376.38 & $0.00 \%$ \\
\hline 500 & $0 .($ & 20 & 1 & 40.82 & 639 & 281.08 & 6400.45 & $0.03 \%$ \\
\hline 500 & 0.02 & 100 & 0.5 & 23.83 & 8917.29 & 125.89 & 8917.31 & $0.00 \%$ \\
\hline 500 & 0.0 & 100 & 1 & 18.01 & 8959.10 & 126.31 & 8941.42 & $0.20 \%$ \\
\hline 500 & 0.0 & 10 & 0.5 & 44.07 & 6061.58 & 472.26 & 6066.87 & $0.09 \%$ \\
\hline 500 & 0.0 & 10 & 1 & 50.49 & 6084.54 & 478.42 & 6090.85 & $0.10 \%$ \\
\hline 500 & 0.0 & 20 & 0.5 & 35.37 & 6396.94 & 284.28 & 6398.14 & $0.02 \%$ \\
\hline 500 & 0.0 & 20 & 1 & 35.50 & 6420.17 & 283.06 & 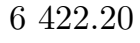 & $0.03 \%$ \\
\hline 500 & 0.0 & 100 & 0.5 & 21.37 & 8929.61 & 131.20 & 8939.28 & $0.11 \%$ \\
\hline 500 & 0.04 & 100 & 1 & 21.18 & 8951.67 & 131.63 & 8963.38 & $0.13 \%$ \\
\hline \multicolumn{4}{|c|}{ Average } & 35.37 & & 284.91 & & $0.07 \%$ \\
\hline
\end{tabular}

Table 1: Performance of the solution algorithm in case of $M=2$ 


\begin{tabular}{|c|c|c|c|c|c|c|c|c|}
\hline \multirow[b]{3}{*}{$\mathrm{a}$} & \multirow[b]{3}{*}{$\mathrm{h}$} & \multirow[b]{3}{*}{$\mathrm{p}$} & \multirow[b]{3}{*}{$\mathrm{w}$} & \multicolumn{5}{|c|}{$\mathrm{M}=3$} \\
\hline & & & & \multicolumn{2}{|c|}{ CS approach } & \multicolumn{2}{|c|}{ SAA Approach } & \multirow[b]{2}{*}{$\%$} \\
\hline & & & & CPUs & E-CS & CPUs & E-SAA & \\
\hline 250 & 0.02 & 10 & 0.5 & 55.84 & 4266.77 & 445.58 & 4273.36 & $0.15 \%$ \\
\hline 250 & 0.02 & 10 & 1 & 54.97 & 4267.57 & 444.91 & 4274.58 & $0.16 \%$ \\
\hline 250 & 0.02 & 20 & 0.5 & 47.55 & 4604.56 & 292.38 & 4602.29 & $0.05 \%$ \\
\hline 250 & 0.02 & 20 & 1 & 51.79 & 4598.56 & 292.78 & 4603.51 & $0.11 \%$ \\
\hline 250 & 0.02 & 100 & 0.5 & 22.27 & 7118.65 & 159.28 & 7115.32 & $0.05 \%$ \\
\hline 250 & 0.02 & 100 & 1 & 23.20 & 7114.27 & 159.04 & 7116.54 & $0.03 \%$ \\
\hline 250 & 0.04 & 10 & 0.5 & 45.14 & 4289.65 & 451.54 & 4297.92 & $0.19 \%$ \\
\hline 250 & 0.04 & 10 & 1 & 46.21 & 4292.36 & 453.91 & 4299.14 & $0.16 \%$ \\
\hline 250 & 0.04 & 20 & 0.5 & 32.27 & 4623.46 & 322.44 & 4622.92 & $0.01 \%$ \\
\hline 250 & 0.04 & 20 & 1 & 31.60 & 4624.95 & 320.81 & 4624.14 & $0.02 \%$ \\
\hline 250 & 0.04 & 100 & 0.5 & 19.76 & 7141.74 & 161.24 & 7139.52 & $0.03 \%$ \\
\hline 250 & 0.04 & 100 & 1 & 20.74 & 7143.25 & 162.05 & 7140.74 & $0.04 \%$ \\
\hline 500 & 0.02 & 10 & 0.5 & 48.60 & 6017.28 & 491.71 & 6023.72 & $0.11 \%$ \\
\hline 500 & 0.02 & 10 & 1 & 42.23 & 6018.43 & 490.53 & 6024.94 & $0.11 \%$ \\
\hline 500 & 0.02 & 20 & 0.5 & 32.26 & 6349.65 & 315.73 & 6349.74 & $0.00 \%$ \\
\hline 500 & 0.02 & 20 & 1 & 34.53 & 6348.71 & 313.72 & 6350.96 & $0.04 \%$ \\
\hline 500 & 0.02 & 100 & 0.5 & 23.70 & 8874.10 & 161.54 & 8867.33 & $0.08 \%$ \\
\hline 500 & 0.02 & 100 & 1 & 18.09 & 8843.12 & 161.43 & 8868.56 & $0.29 \%$ \\
\hline 500 & 0.04 & 10 & 0.5 & 43.59 & 6041.52 & 514.15 & 6046.52 & $0.08 \%$ \\
\hline 500 & 0.04 & 10 & 1 & 46.65 & 6042.33 & 516.14 & 6047.75 & $0.09 \%$ \\
\hline 500 & 0.04 & 20 & 0.5 & 34.15 & 6371.62 & 324.92 & 6378.35 & $0.11 \%$ \\
\hline 500 & 0.04 & 20 & 1 & 35.77 & 6378.56 & 324.59 & 6379.57 & $0.02 \%$ \\
\hline 500 & 0.04 & 100 & 0.5 & 19.39 & 8881.60 & 165.02 & 8891.59 & $0.11 \%$ \\
\hline 500 & 0.04 & 100 & 1 & 19.65 & 8871.92 & 164.53 & 8892.81 & $0.23 \%$ \\
\hline \multicolumn{4}{|c|}{ Average } & 35.41 & & 317.08 & & $0.09 \%$ \\
\hline
\end{tabular}

Table 2: Performance of the solution algorithm in case of $M=3$

We then investigated the impacts of some critical parameters such as ordering cost, unit purchase price, unit shortage cost on the expected total system cost.

Figure 1b) presents the effect of the unit purchase price on the total cost of perishable inventory. The horizontal axis represents the various unit purchase price from $80 \%$ to $130 \%$ in comparison with its base value. The vertical axis shows the total expected system costs under different values of the unit purchase price. This result indicates that with the increase in purchase price, the total system cost will increase rapidly. This confirms the fact that buying cost plays the most crucial role in this system with $72 \%$ of total cost.

Figure 1c) shows that the total system cost grows up slightly as the function of ordering cost, however, this increases is not as significant as the one of ordering cost from $80 \%$ to $130 \%$, in comparison with its base value. Figure 1d) shows that the total system cost seems to be sensitive to unit shortage cost. The horizontal axis represents the various unit shortage cost from $80 \%$ to $130 \%$ in comparison with its base value. The vertical axis shows the total expected system costs under different values of unit shortage cost. 

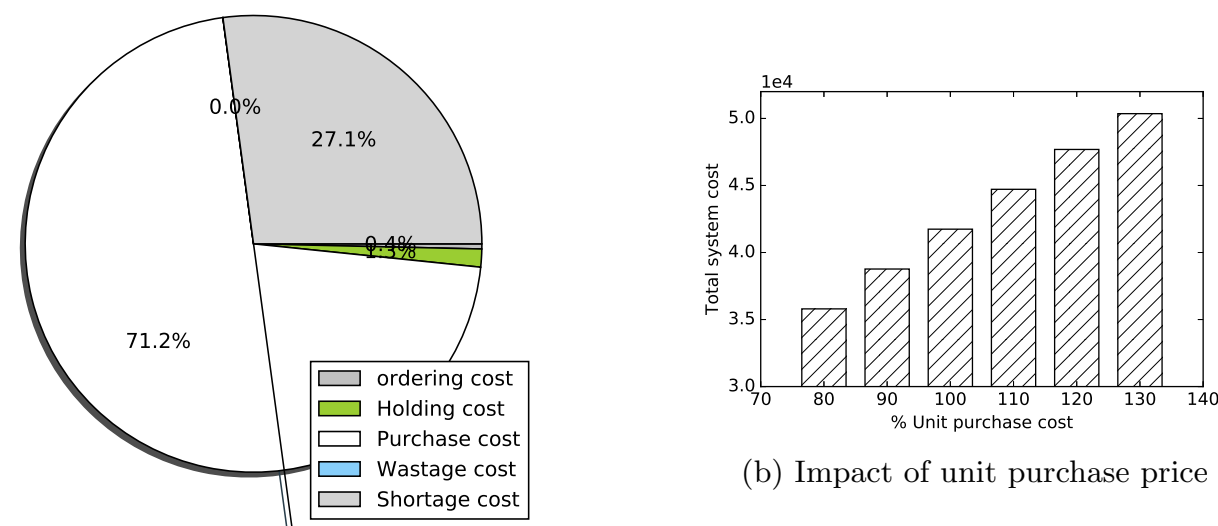

(b) Impact of unit purchase price

(a) Cost distribution of inventory

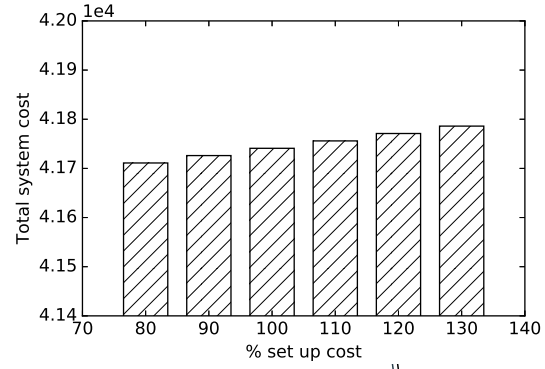

(c) Impact of unit ordering cost

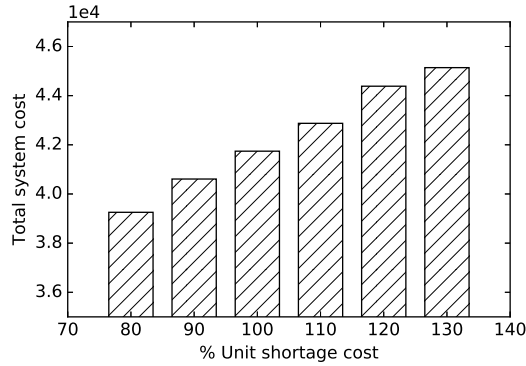

(d) Impact of unit shortage cost

Figure 1: Analysis of the solution

\section{Conclusion}

In this paper, we proposed a stochastic model for minimizing the total cost of a perishable inventory system under stochastic environments in a rolling horizon framework. We also proposed a CS method to solve approximatively the stochastic programming model. The CS approach seems practical since the computation of the conditional scenarios is comprehensible and straightforward. It requires only a set of samplings generated from conditional expectations of random parameters. The CS approach appears a better choice in comparison with the SAA approach since the former can provide a better solution in a shorter computation time.

For future research, other uncertainties (price, quality) and risk management may be considered. Moreover, an effective algorithm is required to cope with the complexity and the high computational challenge of the stochastic inventory problem.

\section{References}

[1] Bakker, M., Riezebos, J. and Teunter, R. H. (2012). Review of inventory systems with deterioration since 2001. European Journal of Operational Research, 221(2), 275-284. doi: 10.1016/j.ejor.2012.03.004

[2] Beltran-Royo, C. (2017). Two-stage stochastic mixed-integer linear programming: The conditional scenario approach. Omega, 70, 31-42. doi: 10.1016/j.omega.2016.08.010 
[3] Broekmeulen, R. A. C. M. and van Donselaar, K. H. (2009). A heuristic to manage perishable inventory with batch ordering, positive lead-times, and time-varying demand. Computers and Operations Research, 36(11), 3013-3018. doi: 10.1016/j.cor.2009.01.017

[4] Diwekar, U. and David, A. (2015). BONUS Algorithm for Large Scale Stochastic Nonlinear Programming Problems. New York: Springer. doi: 10.1007/978-1-4939-2282-6

[5] Janssen, L., Claus, T. and Sauer, J. (2016). Literature review of deteriorating inventory models by key topics from 2012 to 2015. International Journal of Production Economics, 182, 86-112. doi: 10.1016/j.ijpe.2016.08.019

[6] Mckay, M. D., Beckman, R. J. and Conover, W. J. (1979). Comparison of three methods for selecting values of input variables in the analysis of output from a computer code. Technometrics, 21(1), 239-245. doi: 10.1080/00401706.1979.10489755

[7] Pauls-Worm, K. G. J., Hendrix, E. M. T., Alcoba, A. G. and Haijema, R. (2016). Order quantities for perishable inventory control with non-stationary demand and a fill rate constraint. International Journal of Production Economics, 81, 238-246. doi: 10.1016/j.ijpe.2015.10.009

[8] Tajbakhsh, M. M., Zolfaghari, S. and Lee, C. G. (2007). Supply Uncertainty and Diversification: A Review, 345-368. In Jung. H., Jeong, B. and Chen, F. F. (Eds): Trends in Supply Chain Design and Management (Series in Advanced Manufacturing). London: Springer. doi: 10.1007/978-184628-607-0_15

[9] The Food Marketing Institute. (2018). The Collaborative Strategies to Reduce Unsaleables Report. https://www.gmaonline.org/file-manager/TPA_ CollaborativeValueChainStrategiestoReduceUnsaleables.pdf 